\title{
United Kingdom scheme for external quality assessment in virology. Part I. General method of operation
}

\author{
SYLVIA E REED, ${ }^{*}$ PS GARDNER, ${ }^{*}$ JJS SNELL, ${ }^{*}$ OPHELIA CHAI $\dagger$ \\ From the *Division of Microbiological Reagents and Quality Control, Central Public Health Laboratory, \\ Colindale, London NW9, and the †Computer Services, Public Health Laboratory Service Board, Colindale, \\ London NW9
}

SUMMARY Developments in the United Kingdom national external quality assessment scheme for virology are described. There are about 198 participants (170 in the UK) who are enrolled for examination of any or all of five categories of specimen (distribution types). These are detection of rubella antibody (128 UK participants), detection of hepatitis B surface antigen (130 UK participants), general virus serology (86 UK participants), virus identification (85 UK participants), and electron microscopy (56 UK participants). Specimens of a sixth category (rubella IgM antibody), not yet formally established, have also been distributed to 67 UK participants. Specimens in each distribution type are sent out once or twice a year, and, except for rubella IgM antibody, participants have been given a score of $2,1,0$ or -1 marks for their reports on each specimen. Their cumulative scores and performance ratings are calculated retrospectively over a 12 month period for each distribution type separately and for combined distributions. The performance rating is defined by the number of standard errors by which the individual's cumulative score differs from the mean for all participants and carries a + or - sign depending on whether the cumulative score lies above or below the mean. Performance ratings have been found generally to be close to the mean in rubella serology and detection of hepatitis B surface antigen but are more variable in virus identification and electron microscopy. Ratings of $<-1.96$ are considered to be significantly worse than average and to constitute poor performance.

The principles of the United Kingdom national external quality assessment scheme in various pathology disciplines have been reviewed by Whitehead and Woodford ${ }^{\prime}$ and the operation of the scheme for microbiology during 1974-80 has been described elsewhere. ${ }^{2}$ The UK scheme is voluntary but all laboratories concerned with examining specimens relevant to care of patients are encouraged to participate and the rate of compliance appears to be high. Laboratories participating in microbiology receive and report on simulated specimens in a number of categories, of which the largest is general bacteriology (bacterial isolation and identification). Participants are given a score for each of the specimens they examine, and the scores

Accepted for publication 15 January 1985 are reported back to the participants in confidence. In general bacteriology, analysis of six monthly cumulative scores gives each laboratory an assessment of its overall performance and allows definition of laboratories whose performance is significantly worse than average.

In $1975 \mathrm{Hart}^{3}$ described the distribution of simulated specimens for virus isolation or assay of antibodies against influenza and rubella to up to 60 laboratories in the UK. This nucleus of external quality assessment (EQA) in virology was developed further, and in 1980 there were five categories of virology - that is, virus isolation, general virus serology, rubella serology, hepatitis B surface antigen detection, and electron microscopy. ${ }^{2}$ Participants in virology, however, received no individual appraisal of their results, as was the case for general clinical bacteriology. Since 1980 the scheme for EQA in virology has been further extended. 
Improved procedures for preparation and predistribution assessment of specimens have been established, and methods of scoring results have been defined. Computer systems for storage and analysis of records have been developed, giving participants printed records of their scores and cumulative performance ratings. As in general bacteriology, laboratories whose cumulative scores are significantly below average have been identified.

The first intention of the scheme is to provide participating laboratories with well documented virological specimens with which to monitor their work and to evaluate the performance of each laboratory by comparison with others examining the same specimens. As in EQA for general clinical bacteriology, individual laboratories' results, scores, and cumulative performance ratings are strictly confidential: participants' reports are identified by laboratory code numbers and the addresses associated with these codes are available only to relevant members of staff. However, advice from the National Advisory Panel for Microbiology is made available to any laboratories whose performance is significantly worse than average, although the identity of such laboratories is made known to the panel only in exceptional circumstances, if poor performances persists over a prolonged period.

The virology scheme also provides participating laboratories with technical information and perhaps with a means of self education. In addition to reporting on each specimen, participants are asked to give additional details of their results and the methods they have used. Analyses circulated after each distribution of specimens summarise the overall results obtained and the methods reported and provide comment on the difficulties or successes encountered with individual specimens or with various techniques or reagents. Critical use of these summaries may, where relevant, help participants to identify areas of difficulty and perhaps provide guidance on means of improving their performance. Some of the specimens may usefully be retained by participants for reference purposes - for example, a dilution of the British Standard for hepatitis B sur- face antigen or, in the case of electron microscopy, material containing viruses known to be difficult to identify in clinical specimens.

\section{Methods and results}

\section{TYPES OF SPECIMENS DISTRIBUTED}

Five categories of specimen (distribution types) are already documented and a sixth is being created.

\section{Rubella serology}

Serum specimens said to have been provided by "nursing recruits" are distributed for screening for the presence of rubella antibody (IgG), and participants are asked to report on the presence or absence of antibody and the need, or otherwise, for vaccination. Recently, samples containing rubella IgM antibody have also been distributed. These specimens have proved valuable to laboratories introducing newer techniques for detection of IgM.

\section{Hepatitis $B$}

The intention is to monitor performances in detection of hepatitis B surface antigen in human serum samples, and the scoring is based on this. Participants may, if they wish, also use the same specimens to assess tests for other markers of hepatitis B infections, and, although no scores are given for results of these other tests, they are recorded and analysed in the distribution summary.

\section{General virus serology}

Two or more serum samples are distributed for testing by complement fixation against certain viral antigens or Mycoplasma pneumoniae, Coxiella burneti, or the Chlamydia group. Scoring is based on the ratio of the titres of two samples tested against each specified antigen.

\section{Virus identification}

The simulated specimens contain viruses for identification by culture or other methods. This distribution type is closely analogous to general clinical bacteriology. Scoring is based on the accuracy of identification.

Table 1 Distributions and specimens for external quality assessment in virology in 1982 and 1983

\begin{tabular}{|c|c|c|c|c|}
\hline \multirow[t]{2}{*}{ Distribution type } & \multicolumn{2}{|l|}{1982} & \multicolumn{2}{|l|}{1983} \\
\hline & No of distributions & No of specimens & No of distributions & No of specimens \\
\hline $\begin{array}{l}\text { Rubella (IgG)* } \\
\text { Rubella (IgM) } \\
\text { Hepatitis B* } \\
\text { General virus serology* } \\
\text { Virus identification* } \\
\text { Electron microscopy }\end{array}$ & $\begin{array}{l}2 \\
1 \\
2 \\
2 \\
2 \\
1\end{array}$ & $\begin{array}{l}5,4 \\
3 \\
4,6 \\
4,4 \\
3,3 \\
4\end{array}$ & $\begin{array}{l}2 \\
1 \\
1 \\
2 \\
2 \\
1\end{array}$ & $\begin{array}{l}4,5 \\
3 \\
5 \\
3,4 \\
4,3 \\
4\end{array}$ \\
\hline
\end{tabular}

${ }^{*}$ Distributions were made at 6 to 7 month intervals. 
Table 2 Numbers of laboratories in various categories receiving specimens for external quality assessment in virology (March 1983)

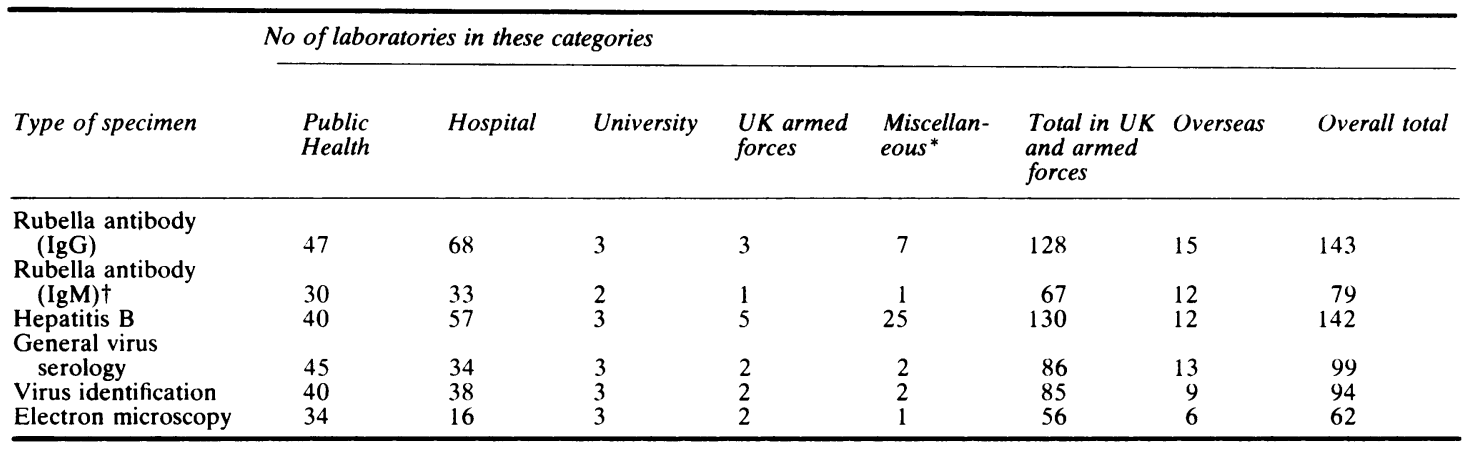

*Includes blood transfusion laboratories.

†At October 1983.

\section{Electron microscopy}

Again, simulated specimens are distributed for identification of viruses and the scores allocated depend on the accuracy of identification.

Sets of specimens in most of these categories are distributed twice yearly, roughly every six to seven months, although only one distribution a year has so far been made for electron microscopy and rubella IgM (Table 1). The limited availability of human

serum samples may restrict the dispatch of specimens, and this applies particularly to samples negative for rubella antibody or containing rubella IgM antibody.

PARTICIPANTS IN THE SCHEME

The scheme is intended mainly for participants in the UK, but a number of non-UK laboratories are also enrolled. For reasons of resources and organisa-

Table 3 Procedures followed in each external quality assessment (EQA) distribution

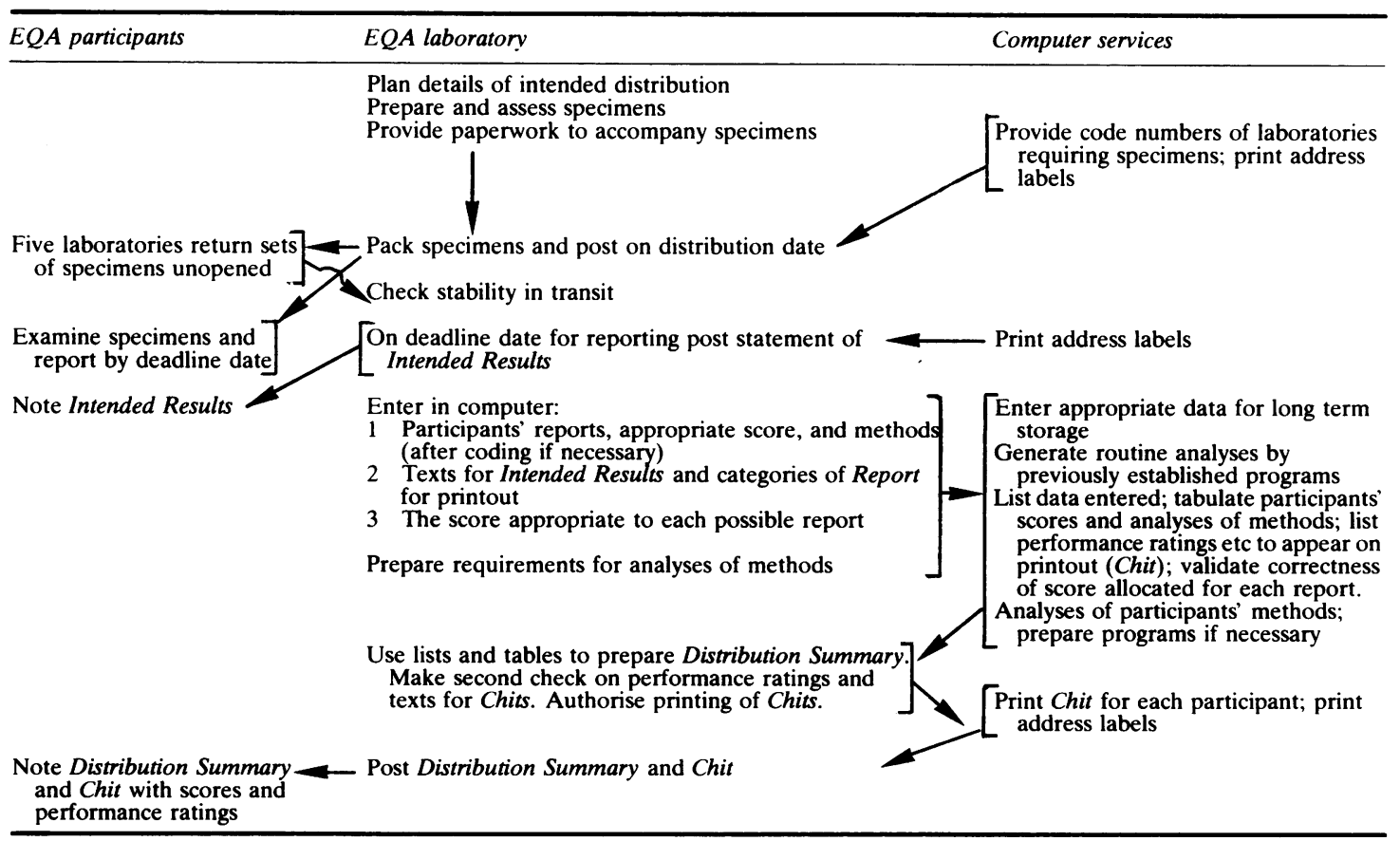

Enter in computer: (after coding if necessary)

Texts for Intended Results and categories of Report for printout

The score appropriate to each possible report

Prepare requirements for analyses of methods

se lists and tables to prepare Distribution Summary. Make second check on performance ratings and

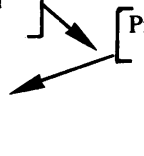


tion the number of such overseas laboratories has recently had to be restricted. UK participants include laboratories in England, Wales, Scotland, Northern Ireland, and the British armed forces laboratories both within the UK and abroad. Overseas laboratories enrolled in the virology scheme are in Eire, Italy, Switzerland, Austria, West Germany, Finland, Saudi Arabia, and South Africa. Postal regulations have prevented dispatch of infectious specimens to certain other countries. The total number of laboratories registered is 198 , of which 170 are UK participants. Table 2 shows the numbers receiving various categories of specimens in 1983 . About $25 \%$ of UK participants take all five of the present distribution types and $32 \%$ take only one type, usually hepatitis or rubella.

\section{LABORATORY AND COMPUTING PROCEDURES} COMMON TO EACH DISTRIBUTION OF SPECIMENS Table 3 outlines the procedure for each distribution of specimens. The EQA laboratory is responsible for preparation of specimens, for pre- and postdistribution assessment of their quality and stability, and for all associated paperwork. This includes preparation of participants' reports and scores for com- puter entry, the texts for computer printout, and specifications for the required analyses of methods. Later, the distribution summary is prepared from computer derived tables. This summary describes participants' reports for each specimen and usually incorporates information about the methods used and the results associated with each method. Comments on points of interest are also included.

The EQA laboratory has required considerable computing resources to develop the system of analysis described. The Computer Services of the Public Health Laboratory Service have provided facilities for maintaining the register of participants and for generating summaries and analyses of the information collected after each distribution of virological specimens. As necessary, new or modified programs have been prepared for analyses of participants' methods. Computing has hitherto been carried out on a Hewlett Packard 9830A microcomputer with $4 \mathrm{~K}$ of random access memory. The operation of the virology EQA scheme, however, is being transferred to a Sirius 1 microcomputer programmed in BASIC. The register of participants in the microbiology EQA scheme is common to all laboratories requiring specimens, both virological

Table 4 Example of data included in the computer printed chits dispatched to each participating laboratory after each distribution of specimens for external quality assessment in virology

\begin{tabular}{|c|c|c|c|c|}
\hline \multicolumn{5}{|c|}{ Microbiology quality assessment scheme } \\
\hline Laboratory no 999 & & Rubella serology distrib & & Date 09/09/99 \\
\hline $\begin{array}{l}\text { Specimen } \\
\text { Intended result } \\
\text { Your report } \\
\text { Your score }\end{array}$ & $\begin{array}{l}123 \\
\text { Positive, immune } \\
\text { Positive, immune } \\
2\end{array}$ & $\begin{array}{c}124 \\
\text { Negative, non-immune } \\
\text { Negative, non-immune } \\
2\end{array}$ & $\begin{array}{c}125 \\
\text { Positive, immune (scoring } \\
\text { category weak pos.) } \\
\text { Negative, non-immune } \\
0\end{array}$ & \\
\hline $\begin{array}{l}\text { Specimen } \\
\text { Intended result } \\
\text { Your report } \\
\text { Your score }\end{array}$ & $\begin{array}{l}126 \\
\text { Positive, immune } \\
\text { Positive, immune } \\
2\end{array}$ & & & \\
\hline
\end{tabular}

Total number of specimens sent to you for rubella serology since 10/09/98-13

Number of reports returned and scored

Number for which no report was received (scored 0) 0
Number of specimens not examined (not scored) 0 Number of reports too late for analysis (not scored) 0

Your cumulative score for the 13 specimens scored for rubella serology was 24 out of a possible total of 26 . The mean score calculated from the performance of all laboratories scored for these same specimens was $24 \cdot 91$, with a standard error of 1.91

Your performance rating for rubella serology (ie, the number of standard errors by which your cumulative score lies above or below the mean) is -0.48

Combined virology distributions

Distribution numbers $292,289,285,281,277,275,267,263,260,257$ have been analysed, covering the 12 month period since $10 / 09 / 98$. For the distributions sent to your laboratory 32 virology specimens were scored.

Number of reports returned and scored $\begin{array}{lr}\text { Number of reports returned and scored } & 31 \\ \text { Number for which no report was received (scored 0) } & 0\end{array}$

Number of specimens not examined (not scored) 1 Number of reports too late for analysis (not scored) 0

Your cumulative score for these 31 specimens was 56 out of a possible total of 62 . The mean score calculated from the performance of all laboratories scored for these same specimens was 55.04 with a standard error of 3.53.

Your performance rating from combined virology distributions (ie, the number of standard errors by which your cumulative score lies above or below the mean) is +0.27 
and otherwise. The information stored includes laboratory code number, with corresponding name and address, laboratory categories (public health, hospital, university, and so on), country of location, and the types of specimen (distribution types) required.

The computer stores the records of all virological specimens dispatched and the results reported by the participants and provides analyses after each distribution and also every six months. After each distribution a computer printout (chit) is printed for each participant (Table 4) which gives the results for each of the specimens within the distribution and the score allocated for each. The chit also records performance ratings calculated from the individual laboratory's scores accumulated over the preceding 12 months. At the end of the calendar year each participant also receives a computer printed list of all the specimens he has been sent during the year, with his report and score for each.

\section{SCORES AND PERFORMANCE RATINGS}

Each specimen examined is given a score on the scale $2,1,0,-1$

Score 2: fully correct

Score 1: partly correct

Score 0: incorrect, but the results reported would not be of extreme clinical importance-for example, false negative test for rubella antibody (IgG); failure to detect influenza virus. Failure of a participant to make any response to dispatch of a specimen (no return) is also recorded 0 .

Score -1 : wrong report which in a clinical or epidemiological context would have serious significance-for example, false positive for hepatitis B surface antigen; reporting adenovirus in error for influenza virus.

Reports received late and the report "specimen not examined" are not scored and the participants are therefore not penalised for these responses. Occasional specimens considered by the quality assessment laboratory to have deteriorated in transit or have proved unsatisfactory in some other way are not scored.

Assessment of participants' cumulative performances resembles that used for EQA in general bacteriology. For virology, however, cumulative scores and performance ratings are calculated for the preceding 12 months, whereas a six month period is used in bacteriology. In virology, laboratories are given one performance rating for the preceding year's specimens within each single distribution type-for example, hepatitis B-and another for combined distributions, comprising all the virological EQA specimens examined by that laboratory in the preceding year. The calculation of the perfor- mance rating is based on the method described by Tillett and Crone. ${ }^{4}$ The rating is the number of standard errors by which the participant's cumulative score for the preceding 12 months differs from the mean, and the mean with which each individual laboratory's performance is compared is calculated from the results of all participants examining the same specimens as the individual concerned. The performance rating carries a + or - sign, indicating whether performance is above or below the mean. The differences from the mean are likely to be significant only if the performance rating is $>+1.96$ or $<-1.96$.

\section{A laboratory's performance rating $(p)$ is}

$\frac{m-r}{\text { standard error of } r}$

where $\mathrm{m}=$ the sum of that laboratory's scores for the specimens submitted and $r=$ the mean score achieved by all laboratories examining those specimens. The mean $(r)$ and its standard error against which the individual's cumulative performance is judged is calculated as shown below for each individual laboratory using the specimen means $(t)$ for all the specimens submitted to that laboratory. Inclusion of all the appropriate specimen means makes allowances for some specimens having proved more difficult than others; a laboratory that has examined mainly difficult specimens is not compared with a mean derived from easy specimens.

Where $f=$ score of each laboratory examining a particular specimen and $w=$ total number of laboratories scored for that specimen (including those scored zero for failing to return a report)

Then $\mathrm{t}=$ specimen mean

$=\frac{\text { sum of } f \text { for all laboratories examining the specimen }}{w}$

and $S^{2}=$ specimen variance $=\frac{\Sigma(f-t)^{2}}{w}$

Then $r=$ the sum of $t$ for all relevant specimens and standard error of $r=0.5+\sqrt{\text { sum of } S^{2} \text { for all relevant specimens }}$

Performance ratings for participants in the UK and British armed forces are derived from means calculated for these participants only, whereas performance ratings for overseas participants are derived from means calculated for all participants (UK, British armed forces, and overseas).

Performance ratings of less than -1.96 are considered to be significantly worse than average and to indicate poor performance in individual or combined distribution types. Performance ratings calculated for the preceding year's specimens may be derived from as few as four specimens (electron microscopy) or as many as $\mathbf{3 0}$ or more (combined distributions), and the numbers of participants concerned may vary from about 55 (electron micros- 
copy) to about 200 . Even with small numbers of specimens and participants, however, as in electron microscopy, the probability that a poor performance cumulative score would occur by chance is low ( $p<$ 0.025 ) and is in good agreement with the exact probability computed from all possible combinations of scores (J Lobb, personal communication). The latter approach requires too much computation to be adopted routinely.

\section{PERIODICAL ASSESSMENTS OF PERFORMANCE}

The Figure shows the distribution of performance ratings for participants in the UK and British armed forces for each distribution type separately and five distribution types combined (combined analysis). For rubella antibody detection and $\mathrm{HBsAg}$ detection most participants' ratings are close to the average, but in general serology, virus identification, and electron microscopy ratings are more widely distributed. In virus identification, electron microscopy, and combined analysis the performance ratings of a number of participants lie between +0.67 and +1.65 standard errors above the mean, and for combined analysis a few performance ratings significantly above the average-that is, $>+1.96-$ have been recorded. The proportion of poor performers - that is, those laboratories with ratings
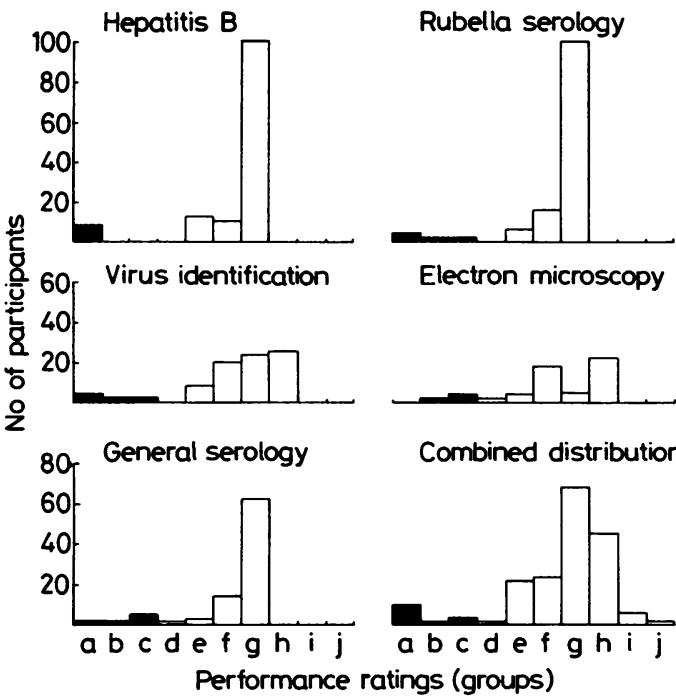

Performance ratings of participants in virology external quality assessment analysed for five separate distribution types and for all distribution types combined. The ratings have been grouped into the following categories; $a<-3 \cdot 3$; $b-3.3$ to $<-2.6 ; c-2.6$ to $<-1.96 ; d-1.96$ to $<-1.65$; $e-1.65$ to $<-0.67 ; f-0.67$ to $<0.0 ; g 0.0$ to $<+0.67 ; h$ 0.67 to $<+1.65 ; i+1.65$ to $<+1.96 ; j \geqslant+1.96$. Ratings of $<1.96$, indicating potential poor performance, are shown as dark columns $<-1.96$-is about $5 \%$ for rubella serology and HBsAg detection and 8 or $9 \%$ for other distribution types and for combined analysis. Poor performance in combined analysis is usually associated with poor performance in one or more of the distribution types analysed singly, but the converse is not always the case. From 1984 it is anticipated that participants with performance ratings $<-1.96$ will be defined by analyses carried out each January and July, each analysis being retrospective for 12 months. The attention of participants whose performance ratings are $<-1.96$ will, in confidence, be drawn to this fact. If poor performance in an individual laboratory should persist a procedure similar to that used in EQA of general bacteriology will be followed and the participant will be offered the advice of the National Advisory Panel for Microbiology.

\section{ANALYSIS OF PARTICIPANTS' METHODS}

A request for participants to report on their methods accompanies all EQA specimens for virology except for general virus serology (complement fixation tests). Information on the methods participants use for each specimen is analysed in the EQA laboratory and reported back to the participants in the distribution summaries. New methods are now being widely introduced in clinical virology, particularly enzyme linked immunosorbent assays for hepatitis and rubella and fluorescent antibody methods in virus identification, and many new reagents and kits are being marketed. Distribution summaries record the numbers of participants currently using various techniques and give the success rates associated with each method. Details such as titres or other quantitative results that have been reported for individual specimens are also included in the summary together with any comments thought likely to be of interest and value to those wishing to assess the possible advantages and disadvantages of different techniques. Some results of such analyses are described in the accompanying paper ( $p$ 542).

\section{Discussion}

The importance of EQA is by now well accepted in several disciplines of clinical pathology, especially clinical chemistry. In microbiology, the problems of producing stable, replicate specimens have had to be overcome and scoring schemes have needed to take into account the fact that laboratory reports are usually not based on an absolute quantitative result. The UK scheme for general bacteriology is successfully established, however, with recent developments in relation to antibiotic sensitivity

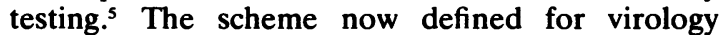
includes detection of $\mathrm{HBsAg}$ and of rubella anti- 
body, tests of major clinical importance each undertaken by about 130 United Kingdom laboratories, as well as virus identification, general virus serology, and electron microscopy.

The establishment of EQA in these five subsections of virology is appropriate to the $\mathrm{UK}$, where all these types of work are often done within one virology laboratory. In contrast, in the USA the Proficiency Testing Program of the US Department of Health and Human Services (Centers for Disease Control, Atlanta) covers hepatitis $\mathrm{B}^{6}$ allied with a range of other serological tests involving bacteria, viruses, protozoa, and fungi.' Also, the College of American Pathologists organises a major series of quality assessment programmes which include both assessment of performance and surveys of methods in various disciplines. Tests for viral hepatitis markers and detection of antibodies to rubella are included but not virus identification or diagnostic electron microscopy. ${ }^{8-10}$

One of the major problems in developing the UK scheme for EQA in virology has been to devise a common framework for allocation of scores and performance ratings and for analyses of methods that can be applied to each of the current five distribution types and, it is hoped, to any new distribution types envisaged. This framework is outlined here and in the accompanying paper ( $p$ 542) and it forms the basis of new computer programs being developed to improve the operation of the scheme.

The allocation of scores of $2,1,0$, or -1 for each specimen has been well accepted by participants, as have the performance ratings in individual and combined distribution types. It has been evident that the receipt of their chits (Table 4 ) has focused the attention of participants on the extent to which their own performance is above or below average and has helped them to identify problem areas which may include clerical or administrative deficiencies. Participants are advised to treat all EQA specimens as if they were routine specimens from patients, but this advice is not always followed and it is probable that performance ratings in EQA often represent a laboratory's best rather than its average work. But the virology EQA scheme should, perhaps over a period of months or years, improve the overall standard of work. Such an improvement might be documented as a change in the distribution of performance ratings (Figure) or as an increase in the overall mean score (specimen mean) for difficult categories of specimen for which the mean scores are sometimes low (p 542). Decreasing use of older, less satisfactory methods may also be documented, with a corresponding increase in newer and more accurate techniques.

The secondary role of the virology EQA scheme in assessing the use of various virological techniques and the relation between each technique and the results reported assumes particular importance because in the UK there is no national system for monitoring the standard or controlling the quality of diagnostic reagents for microbiology. In the absence of such controls careful assessments of the merits and demerits of various techniques, kits, and reagents are necessary. An analysis produced by the EQA scheme may show, for example that 20 laboratories using kit $\mathrm{A}$ for a particular specimen obtained a significantly higher proportion of correct results than 20 other laboratories using kit B and these results may have important implications for the merits of the two kits. It must also be remembered, however, that the two groups of laboratories concerned are not necessarily matched for their technical abilities and that this and other variables may also need to be considered. In other instances the number of users of a particular method may be so small or the variations of technique so numerous that valid statistical analysis is not possible, and comparisons can only provide suggestive rather than conclusive results. The varying needs of different types of laboratory must also be considered when reporting on the performance of various techniques and reagents. For example, blood transfusion laboratories require a highly sensitive technique when testing for hepatitis B surface antigen, whereas a routine clinical laboratory may in certain circumstances require a test in which high specificity and sensitivity may be sacrificed for the sake of speed, simplicity, and low cost. Thus for various reasons data obtained from the EQA distributions on use of various techniques and the results obtained by each method require careful evaluation, as does the manner in which these data are reported back to the participants in the distribution summary. With these provisos the scheme can undoubtedly be valuable in indicating to participants the general trends in methodology and the possible advantages and disadvantages of different modifications of technique.

We thank John Lobb, Communicable Diseases Surveillance Centre, Public Health Laboratory Service, for advice on statistical analyses.

\section{References}

' Whitehead TP, Woodford FP. External quality assessment of clinical laboratories in the United Kingdom. J Clin Pathol 1981;34:947-57.

${ }^{2}$ Snell JJS, DeMello JV, Gardner PS. The United Kingdom national microbiological quality assessment scheme. $J$ Clin Pathol 1982;35:82-93.

${ }^{3}$ Hart RJC. Quality control in diagnostic virology. Proc $R$ Soc Med 1975;68:622-4. 
${ }^{4}$ Tillett HE, Crone PB. Quality control of the isolation rate of pathogens in medical microbiology laboratories.J Hyg (Camb) 1976;77:359-67.

s Snell JJS, Brown DFJ, Gardner PS. Comparison of results from two antibiotic susceptibility testing trials that formed part of the United Kingdom national external quality assessment scheme. J Clin Pathol 1984;37:321-8.

- Taylor RN, Fulford KM. Results of the Center for Disease Control proficiency testing program for the detection of hepatitis B surface antigen. J Clin Microbiol 1976;4:32-9.

'Taylor RN, Fulford KM, Przybyszewski VA, Pope V. Center for Disease Control Diagnostic Immunology Proficiency Testing Program results for 1978. J Clin Microbiol 1979;10:805-14.

${ }^{8}$ Batsakis JG, Lawson NS. Introduction to the report on the quality assurance programs of the College of American Pathol- ogists 1983. Am J Clin Pathol 1983;80 (suppl):551-3.

' Hanson MR, Polesky HF. Radioimmunoassay and enzyme immunoassay methods for detecting viral hepatitis markers. Am J Clin Pathol 1983;80 (suppl):590-3.

${ }^{10}$ Skendzel LP, Wilcox KR, Edson DC. Evaluation of assays for the detection of antibodies to rubella: a report based on data from the College of American Pathologists surveys of 1982. Am J Clin Pathol 1983;80 (suppl):594-8.

Requests for reprints to: JJS Snell, Division of Microbiological Reagents and Quality Control, Central Public Health Laboratory, 61 Colindale Avenue, London NW9 5HT, England. 